Documentación de las Ciencias de la Información ISSN: 0210-4210

http://dx.doi.org/10.5209/DCIN.61407

\title{
Proceso de digitalización del servicio exterior, de México y España: Retos y oportunidades
}

\author{
Laura Romero Vara ${ }^{1}$
}

Envío: 27 de agosto de 2018 / Aceptado: 28 de septiembre de 2018

Resumen. En lo que respecta a la diplomacia, los últimos veinte años se han caracterizado por cambios drásticos en las formas y desarrollo de las relaciones internacionales entre Estados-Nación, principalmente debido a la globalización, el surgimiento de nuevos actores internacionales y por el auge tecnológico en el campo de estudio. Las cancillerías de exteriores han incorporado, como prioridad en sus agendas, la implementación de las nuevas tecnologías a sus programas y objetivos. El objetivo del presente estudio es analizar el proceso de digitalización de los servicios de exteriores de México y de España, los desafíos y los beneficios que tiene para ambos países la digitalización de sus servicios exteriores. También se examinarán las estrategias particulares que se han puesto en marcha y los resultados que se han obtenido a raíz de éstas. Por lo tanto, es interesante realizar este estudio debido a que conociendo en qué punto se encuentra dicha digitalización se facilitara la mejora y agilización de ésta en ambos países.

Palabras clave: Diplomacia digital; Tecnologías de la Información; Digitalización; Servicio Exterior; México; España.

\section{[en] Digitalization process of the foreign service of Mexico and Spain: Challenges and opportunities}

\begin{abstract}
With respect to diplomacy, the last twenty years have been characterized by drastic changes in the forms and development of international relations between nation-states, mainly due to globalization, the emergence of new international actors and the technological boom in the field of study. The foreign chancelleries have incorporated as a priority in their agendas the implementation of modern technologies to their programs and objectives. The objective of this study is to analyze the digitalization process of the foreign services of Mexico and Spain, the challenges and benefits that the digitalization of their external services has for both countries. It will also examine the strategies that have been put in practice and the results that have been obtained as a result of these. Therefore, it is interesting to carry out this study because knowing where this digitalization is will facilitate the improvement and streamlining of this in both countries.
\end{abstract}

Keywords: Digital diplomacy; Information and Communication Technologies; Digitalization; Foreign Service; Mexico; Spain.

Sumario. 1. Introducción y estado de la cuestión. 2. Objetivos y metodología. 3. Diplomacia digital española. 4. Diplomacia digital mexicana. 5. Comparativa de los procesos de digitalización de España y México. 6. Conclusiones. 7. Referencias bibliográficas.

1 CONACYT (Consejo Nacional de Ciencia y Tecnología de México)

lau.rv90@gmail.com 
Cómo citar. Romero Vara, L. (2018) Proceso de digitalización del servicio exterior, de México y España: Retos y oportunidades en Documentación de las Ciencias de la Información 41, 163-182.

\section{Introducción}

El mundo se encuentra inmerso en una era digital, en la cual es indispensable la digitalización, es decir, un proceso de cambio tecnológico, un salto de lo analógico a lo digital en todos los aspectos de la vida de las sociedades del siglo XXI. Las empresas, los gobiernos, y la población en general están haciendo un tremendo esfuerzo por sobrevivir a este nuevo y cambiante entorno digital.

En este sentido, en la última década, los servicios exteriores de casi todos los Estados-Nación, han iniciado su propio proceso de digitalización con el objetivo principal de fortalecer uno de los pilares básicos de la diplomacia: la promoción de los países para incentivar la inversión extranjera y fomentar el turismo, esencialmente a través de la comunicación.

Los cuerpos diplomáticos de una gran mayoría de países avanzan apresuradamente hacia la digitalización para crear una reputación positiva de la "marca país" tanto a nivel nacional como internacional. Hoy más que nunca las TIC son de vital importancia para el desarrollo de la diplomacia. Las redes sociales son una herramienta esencial para aumentar la transparencia, amplificar los mensajes y la rapidez con la que éstos llegan al público reduciendo costos.

Tal y como explica el exembajador australiano en Israel, Sharma (2015), "la tecnología ha irrumpido en la profesión de la diplomacia. Los monopolios naturales han sido destruidos. Los márgenes de ventaja han sido erosionados. La diplomacia en estos días es un espacio mucho más disputado y competitivo".

Diplomáticos y think tanks como Arturo Surukham, Nicholas Westcott, Manfredi Sanchez o Ilan Manor coinciden en que la diplomacia digital no es un nuevo tipo de diplomacia, por el contrario, es una extensión de la diplomacia pública que utiliza las herramientas tecnológicas para alcanzar sus objetivos.

\subsection{Proceso de digitalización diplomática}

Probablemente el principal reto que enfrentan los países a la hora de buscar una diplomacia digital sea la renuencia de muchos diplomáticos a dar el salto de lo privado a lo público, ya que esta profesión se ha caracterizado por ser, en gran parte, cerrada hasta hace unos cuantos años. Hoy en día, la diplomacia tradicional ha quedado relegada, "planteándose acciones en el exterior más allá de las simples relaciones para alcanzar a la opinión pública y publicada internacionales" Borau, et al (2012: 80).

La falta de formación en materia digital del personal de los ministerios o secretarias retrasa el proceso de digitalización de las instituciones gubernamentales. De acuerdo con Rodríguez (2015) es necesario contar con agentes de la diplomacia, expertos en múltiples asuntos, con una preparación orientada a las necesidades del mundo digital.

A su vez, los servicios exteriores se encuentran, con frecuencia, con falta de recursos económicos para llevar a cabo la modernización de los sistemas que se re- 
quiere para alcanzar la digitalización. Además, los profesionales de la diplomacia se ven obligados a crear contenidos personalizados, innovadores y atractivos para un público variopinto para alcanzar el tan codiciado engagement ${ }^{2}$.

En general, los inicios de los procesos de digitalización de los servicios exteriores comenzaron por la modernización de sus páginas web, optimizando su lenguaje, facilitando el acceso de los usuarios, incrementando la dinamización con el objetivo principal de incentivar la interacción entre y con el público. La traducción en varios idiomas de las webs principales de los ministerios y/o secretarias de exteriores, de las embajadas y consulados es fundamental para amplificar los mensajes y la información dirigidos tanto a nacionales como extranjeros.

Aunado a la profesionalización de las páginas webs, las cancillerías de exteriores, sus respectivas embajadas y consulados y los propios ministros de exteriores y trabajadores han tenido que consolidar su presencia en diversas redes sociales. La favorita de muchos, hasta ahora, es Twitter, aunque se valora la presencia en Facebook, Instagram, YouTube entre otras, "los líderes internacionales han ido incursionado y aplicado la diplomacia digital, utilizando como su principal red social Twitter" Banda (2015).

De acuerdo con los últimos datos recogidos en el estudio Twiplomacy (2018) por la empresa Burson-Marsteller, hay 951 cuentas de Twitter que pertenecen a cabezas de Estado, gobiernos, y ministros de exterior en 193 países, representando al 97\% de los Estados Miembro de la ONU, combinados tienen una audiencia de aproximadamente 356 millones de seguidores.

Los gobiernos buscan alcanzar la fase más alta de madurez en cuanto a digitalización diplomática, tanto en las necesidades de capacidad (presencia, personalización y actualización) como en las necesidades de competencia (interacción, influencia y autorrealización ${ }^{3}$ ) para desarrollar relaciones a largo plazo con poblaciones extranjeras y de esta forma alcanzar sus metas.

Pese a la inminente evolución en el ámbito de la penetración de la tecnología en las relaciones internacionales y diplomáticas, aún son muchos los retos que hay que superar para lograr alcanzar una diplomacia $100 \%$ digitalizada y moderna.

\subsection{Casos de éxito}

La puesta en práctica de ciertas acciones, descritas a continuación, por parte de EE.UU., Reino Unido y Francia, los ha convertido en pioneros en el proceso de digitalización.

2 Se entiende por engagement la capacidad de un producto (una marca, un blog, una aplicación, persona o institución) de interaccionar con sus usuarios, creando vínculos permanentes que impulsen a estos a tomar decisiones en pro del producto con él que se establece dicha relación, Parra (s/n)

3 "La Diplomacia digital es la etapa de "autorrealización", que logra el pleno potencial de los ministerios de relaciones exteriores, incluidas las soluciones creativas e innovadoras. El nombramiento de un embajador digital, el diseño de la estrategia y las directrices de diplomacia digital, la creación de un juego para dispositivos móviles o la aplicación progresiva son todos ejemplos perfectos de los objetivos de diplomacia digital definitiva que se espera que logre cualquier AMF." Traducción propia. Recuperado en 25 de julio de 2018 de http://digital. diplomacy.live/methodology-scope/ 


\subsubsection{La controvertida diplomacia digital de EE.UU}

De acuerdo con el estudio Atlas de la Diplomacia Digital 2017, realizado por el Centro de Estambul para Asuntos Internacionales, y con el estudio Twiplomacy 2018, elaborado por la empresa Burston-Marsteller, EE.UU figura en primer lugar en cuanto a diplomacia digital y sus líderes figuran dentro de las primeras posiciones en cuanto a número de seguidores e influenciabilidad.

Los inicios de la diplomacia digital de EE.UU tienen lugar alrededor del concepto Diplomacia Transformacional: avivar la política y el servicio exterior estadounidense. En 2006 surge el proyecto "Diplopedia" Bronk (2010), se trata de en un sitio web en el cual los usuarios (en este caso personal de las agencias de relaciones exteriores de EE.UU) crean, comparten y modifican contenidos relacionados con información útil para los trabajadores del servicio exterior. Recomendaciones dirigidas a funcionarios de dicho servicio exterior, expertos en ciertas áreas o países.

Posteriormente, la exsecretaria de Estado, Hilary Clinton continuó con el proyecto bajo la visión del "Poder Inteligente Diplomático" incorporando el uso de tecnologías comerciales como Twitter, Facebook y YouTube, Lee (2009).

En 2017, el Departamento de Estado de los Estados Unidos de América ya administraba 300 cuentas de Twitter, 400 páginas de Facebook y tenía 180 canales de YouTube con la finalidad de perseguir sus objetivos diplomáticos. Actualmente tiene 5 millones de seguidores en Twitter, 1 millón de "me gusta" en su página de Facebook y $230 \mathrm{~K}$ seguidores en Instagram.

La llegada de Donald Trump a la presidencia en 2017 ha tenido un impacto incuestionable en Twitter. El tono del discurso ha cambiado drásticamente. El presidente estadounidense constantemente publica tweets insultando y ridiculizando a sus homólogos en cualquier rincón del planeta, lo cual, a pesar de ser diplomáticamente incorrecto, tiene un alto grado de repercusión a nivel mundial y aumenta las conversaciones acerca de este personaje, y por ende de EE.UU. en la sociedad internacional.

En síntesis, el gobierno estadounidense ha sabido transformar el uso de las tecnologías de la información en provecho de la diplomacia digital "para facilitar la colaboración y la interconexión de personas e información" Murray (2015). La información fluye instantáneamente, ampliando la cantidad de receptores y minimizando los costos.

\subsubsection{Los reyes de la innovación: diplomacia digital del Reino Unido}

Por su parte, Reino Unido, quien fue líder en el Ranking Global de 2016 de Diplomacia Digital, cayó un lugar en el Ranking Global de 2017, probablemente porque no ha avanzado en la digitalización diplomática tan eficientemente como Estados Unidos. Aun así, es un referente a nivel mundial en lo que respecta a diplomacia digital.

En 2008 la Oficina de Exteriores inglesa (Foreign and Commonwealth Office FCO) lanzó una página web que busca reunir todos los sitios de la embajada y alto comisionado de exteriores en la misma plataforma, tal y como expresó el entonces director de comunicaciones, "el sitio web coloca al FCO a la vanguardia de la innovación web y por delante de otros ministerios de relaciones exteriores" Cartmell (2008). Este sitio contaba con contenido actualizado e incluía elementos dinámicos, los cuales fueron muy novedosos en esa época. 
Actualmente, este país cuenta con más de 700 perfiles oficiales en distintas plataformas de redes sociales con poco más de 7 millones de seguidores y ha sido pionero en el uso de redes sociales. Por ejemplo, "el primer ministerio de exteriores en tener presencia en Snapchat, los primero en abrir una cuenta de Twitter de servicio al cliente para dar consejos para viajar. La embajada de Reino Unido en EE.UU. fue la primera en estar en Buzzfeed y la de Nueva Zelanda, en usar Periscope. Fué el primer país en dar cobertura a las visitas reales, vía WhatsApp, y también en retransmitir en Facebook Live", Elliot (2017).

Los puntos clave del éxito de la diplomacia digital de Reino Unido son: asumir riesgos, innovación y mentalidad multimedia buscando la diversidad de orígenes; así como la formación de diplomáticos, fortaleciendo su trabajo en equipo.

Al igual que muchos gobiernos, la Oficina de Exteriores y Commonwealth ha desarrollado una guía para el uso de las redes sociales ${ }^{4}$ en la que se estimula a los diplomáticos a reinventar la aplicación de la diplomacia digital.

\subsubsection{La diplomacia de influencia francesa}

La diplomacia digital francesa tiene su origen en 1995, cuando el Ministerio de Asuntos Exteriores Francés crea su primera su página web. Actualmente recibe 1,7 millones de visitas al mes. Desde el 2009 el ministerio creó su cuenta de Twitter y, a partir del 2012 ha creado sesiones de "preguntas y respuestas que se realizan periódicamente en Twitter y Facebook" France Diplomatie (2017), lo que permite interactuar con los usuarios y aumentar el engagement.

Como parte del proceso de digitalización del servicio exterior francés, éste tiene presencia en diversas plataformas y en distintos lenguajes, ampliando su influencia a nivel internacional. La estrategia de comunicación diplomática digital de Francia ha puesto en práctica un conjunto de acciones para reforzar el diálogo y mejorar la red de comunicación de las misiones diplomáticas. Dentro de estas acciones destaca "el rediseño y animación editorial de la página web en 2013, la creación y sustitución de contenidos, el esfuerzo realizado por liberar datos públicos, la producción de infografías que faciliten el entendimiento de las acciones llevadas a cabo por el ministerio, la creación de un equipo de soporte técnico, editorial y de capacitación", Chounet-Cambas (2014).

Todas las acciones y estrategias, en cuanto a digitalización diplomática se refiere, le permiten al servicio exterior francés ser un ejemplo para los demás países y posicionarse en el tercer lugar del Ranking Global 2017 en Diplomacia Digital.

\subsubsection{La Tecno Diplomacia danesa}

Finalmente, vale la pena mencionar el caso danés. A pesar de que la diplomacia digital danesa ocupa el puesto décimo octavo, y en concreto, la cuenta de Twitter del Ministerio de Asuntos Exteriores, el puesto décimo noveno dentro de los cincuenta lideres mejor conectados del mundo en 2018. El gobierno danés ha logrado hacer que el concepto de diplomacia digital evolucione a pasos agigantados hacia la diplomacia tecnológica.

4 Disponible en: https://www.gov.uk/government/organisations/foreign-commonwealth-office/about/social-media-use 
A finales del año 2017, Dinamarca ha creado la primera embajada tecnológica en tres zonas horarias diferentes: Silicon Valley, Copenhague y Beijín reconociendo, de esta manera, el papel trascendental que juega la tecnología y la digitalización en la resolución de los principales retos globales y su influencia en la área política y diplomática. Hoy en día, no es posible entender las relaciones internacionales como las relaciones entre Estados-Nación, sino que también incluyen relaciones con ciudadanos, gobiernos locales, empresas, así como la industria de la tecnología.

No se descarta que, en breve, más gobiernos sigan el ejemplo danés y se suban al tren de la diplomacia tecnológica.

\section{Objetivos y Metodología}

El objetivo principal que se pretende obtener con esta investigación es definir y describir la situación actual del proceso de digitalización de las cancillerías de exteriores de España y México.

Este trabajo de investigación gira entorno a la hipótesis central de que, pese a que España y México han comenzado su proceso de digitalización en sus servicios exteriores, éstos carecen de profesionalidad y pautas de actuación unificadas.

El presente estudio se plantea en tres fases. La primera consiste en la revisión bibliográfica de revistas científicas e investigaciones de académicos y profesionales de la diplomacia, de esta forma se obtiene una visión clara de las nuevas tendencias diplomáticas y del surgimiento de nuevos actores que demandan mayor participación en la arena internacional.

En la segunda fase se exponen y analizan las medidas específicas de los proyectos de digitalización de España y de México, este punto trata de dimensionar y comparar la actividad digital de la diplomacia de los Estados español y mexicano, enfatizando sus fortalezas y debilidades.

La tercera fase consta de un análisis exhaustivo del grado de comunicación e interacción que pueden tener los usuarios con el Ministerio de Asuntos Exteriores, Unión Europea y Cooperación de España (MAEC) y de la Secretaria de Relaciones Exteriores de México (SRE); tanto en sus páginas web como en blogs y redes sociales habilitadas para dicho propósito.

Para esta etapa se ha realizado entre el 18 y 24 de junio y entre el 2 y 8 de julio del 2018 un seguimiento exhaustivo de las cuentas de redes sociales que los cuerpos diplomáticos (consulados, embajadas, el MAEC y la SRE) de España y México tienen alrededor del mundo. La muestra base del presente análisis fue un total de 100 cuentas de Twitter, Facebook, Instagram y YouTube, siendo una muestra intencional ya que se buscó que las cuentas estudiadas pertenecieran al servicio exterior español y mexicano ubicado en diferentes partes del mundo, de tal forma que se pudiera observar si estos comportaban igual si estaban en lugares desarrollados como Europa o Norteamérica o en los menos desarrollados como seria el caso de países africanos.

Con la suma del seguimiento a las cuentas y la ayuda de la herramienta Twito$n o m y^{5}$ (para el análisis de las cuentas de Twitter) se logró extraer información valiosa

Es una herramienta web que permite examinar una cuenta de Twitter, aportando información relacionada con los tweets, retweets, respuestas, menciones y hashtags. Presenta la información de forma descriptiva y gráfica. http://www.twitonomy.com 
acerca de la presencia en redes sociales, número de seguidores, interacción con los mismos, tipo y frecuencia de publicaciones que realizan, etc.

Se ha elegido analizar los procesos de digitalización diplomática de España y de México para ejemplificar las acciones que se han llevado a cabo dentro de los países iberoamericanos. De acuerdo con los estudios Global Ranking de Diplomacia Digital del 2016 y 2017 realizados por el Centro de Estambul para Asuntos Exteriores, en los cuales se califica el grado de preparación de la diplomacia digital para los ministerios de relaciones exteriores, ambos países se sitúan entre las primeras posiciones de Iberoamérica del ranking.

A partir de la presente investigación, será posible trabajar en crear planes y estrategias de comunicación política identificando cuáles son los canales o las redes sociales más utilizadas, las que más impactan en la sociedad, las emergentes e inclusive, los distintos usos que se le pueden dar a cada una de ellas perfeccionando, de esta manera, los procesos de digitalización de España y México. Esto podrá servir, a su vez, como ejemplo para otras naciones iberoamericanas y del mundo.

\section{Diplomacia Digital Española}

España comenzó su andadura en la diplomacia digital en 2012, ante la inminente necesidad de transmitir en el extranjero, sobre todo, lo que realmente significa la Marca España. Cuando a finales del 2011, el gobierno del expresidente Mariano Rajoy llega a la Moncloa, con el objetivo de limpiar la reputación de España, los esfuerzos se centran en comunicar a la comunidad internacional la determinación de ponerle fin a la devastadora crisis económica que asolaba al país desde el 2008, Rodríguez (2012). Por tal razón se lanza el "Plan de Comunicación de las Misiones Diplomáticas, Representaciones Permanentes y Oficinas Consulares de España en el Exterior”.

Alberto Antón Cortes, el exembajador en Misión Especial para la Diplomacia Digital relata en su artículo "La Diplomacia Española ante el Reto Digital" los inicios de la digitalización del Ministerio de Exteriores español y de sus misiones diplomáticas alrededor del mundo.

El primer desafío fue "de carácter técnico, relacionado con la necesidad de preservar la seguridad de las comunicaciones diplomáticas" Cortes (2016). Una vez solucionado este problema, las representaciones españolas comenzaron a tener presencia en redes sociales. Actualmente (julio, 2018), España cuenta con: 159 cuentas de Twitter, 98 de Facebook, 25 de Instagram, 10 de YouTube, 1 en Weibo y 1 en VK, controladas por sus diferentes representaciones en el exterior. Además, posee un conjunto de blogs con los cuales, se pretende dar a conocer las acciones que se realizan en el exterior desde un punto de vista más cercano y humano, ya que éstos son escritos por los propios funcionarios que trabajan en las representaciones ubicadas alrededor del mundo.

Una fortaleza indiscutible del proceso de digitalización del servicio exterior español es que, en la página del Ministerio de Asuntos Exteriores y Cooperación (MAEC), es posible encontrar los enlaces a las diferentes cuentas de redes sociales de las representaciones, es decir, éstas están organizadas y se sabe, con exactitud, con qué activos digitales cuentan. Ver tabla 1 


\begin{tabular}{|l|c|c|c|c|c|c|}
\cline { 2 - 9 } & \multicolumn{6}{|c|}{ REDES SOCIALES } \\
\cline { 2 - 8 } & $\mathbf{3}$ & $\mathbf{f}$ & $\mathbf{O}$ & You & $\mathbf{6}$ & $\mathbf{w}$ \\
\hline MAEC & 2 & 1 & 1 & 1 & & \\
\hline Embajadas & 104 & 72 & 19 & 7 & & 1 \\
\hline Consulados & 43 & 21 & 3 & 1 & 1 & \\
\hline $\begin{array}{l}\text { Representaciones } \\
\text { Permanentes }\end{array}$ & 9 & 4 & 2 & 1 & & \\
\hline Total & 158 & 98 & 25 & 10 & 1 & 1 \\
\hline
\end{tabular}

Tabla 1. Presencia en redes sociales del servicio exterior español. Fuente: elaboración propia a partir del Atlas de Redes Sociales del MAEC.

A su vez, el MAEC ha hecho públicas un conjunto de normas relacionadas con el uso de las cuentas de Twitter y Facebook ${ }^{6}$ de las representaciones de España en el exterior. Se pretende que los usuarios utilicen un lenguaje cordial y se les hace ver que sus comentarios y opiniones son muy valiosos y se toman en cuenta. Aunque, no siempre será posible recibir una respuesta a ellos. El hecho de que estas normas estén disponibles para todo el público garantiza la transparencia del proceso de digitalización permitiendo, a su vez, a los usuarios conocer, con anterioridad, lo que pueden esperar y deben exigir a sus gobernantes.
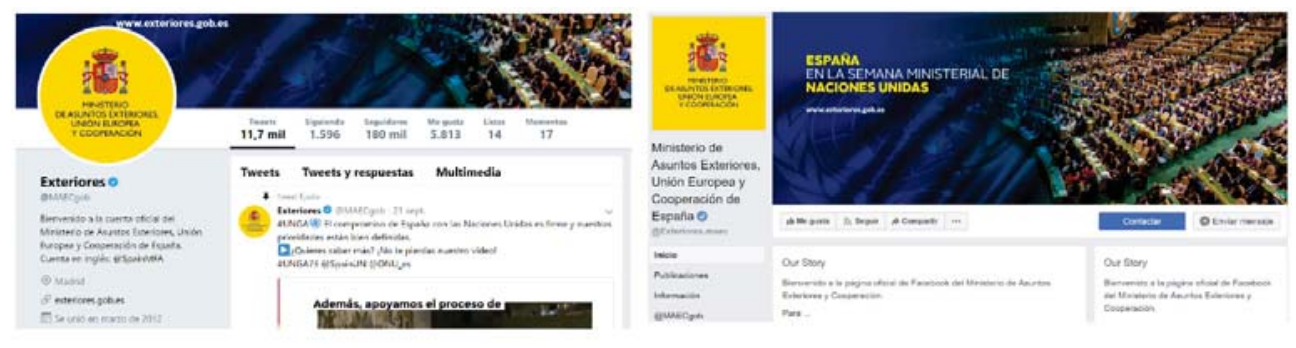

Imagen 1: Perfiles de las cuentas de Twitter (izquierda) y Facebook (derecha) del MAEC. Fuente: cuentas de Twitter y Facebook del MAEC.

Otro reto importante que se le presenta al servicio exterior español en su proceso de digitalización, consiste en preparar a sus funcionarios para la correcta utilización de las nuevas herramientas digitales, especialmente, desde una perspectiva comunicativa. Un gran número de diplomáticos se han sumado al uso de las redes sociales, especialmente Twitter, sin embargo, el cónsul general de España en Jerusalén, Escobar (2016) reconoce que la gran mayoría lo utilizan para informarse de lo que sucede en el mundo, y los pocos que tuitean evitan identificarse como diplomáticos, haciéndolo a título personal.

6 Consulta las normas de uso de las cuentas de Twitter y Facebook del MAEC y las representaciones de España en el exterior en: http://www.exteriores.gob.es/Portal/es/SalaDePrensa/RedesSociales/Documents/NORMAS\%20 USO $\% 20$ TWITTER\%20Y\%20FACEBOOK_2.pdf 
A su vez es importante recordar la necesidad de modernización de los procesos y métodos de trabajo del propio Ministerio, "el servicio diplomático español se ha contado entre los más conservadores de Europa, y de los menos eficaces en la adopción de nuevas maneras de hacer diplomacia." Riordan (2005: 8)

La intención del servicio exterior español es ser capaces de utilizar las oportunidades que brinda el uso de la web y las redes sociales para comunicarse, demostrando cercanía, humanismo y empatía. Benedicto (2016) propone que es necesario una cultura ciber diplomática interactiva y personalizada. El valor agregado no es estar presente en las redes sociales, es comunicar mensajes, pero, sobre todo, escuchar las necesidades del público, buscando el dialogo y el entendimiento.

En este sentido, la autora del presente estudio se ha permitido enviar preguntas y/o comentarios aleatorios a diferentes páginas de Facebook, Twitter, YouTube e Instagram del MAEC y de embajadas y consulados españoles en el exterior escogidas al azar, tratando de abarcar todas las zonas geográficas del globo. En total, se ha tratado de interactuar con 17 perfiles de Facebook, 7 de Twitter, 3 de YouTube y 2 de Instagram. (Ver tabla 2)

Como resultado de este ejercicio, se ha descubierto una falta de homologación de los procesos, métodos de trabajo y responsables de las redes sociales por parte del MAEC, las embajadas y los consulados españoles alrededor del mundo.

Por ejemplo, en el caso de Facebook, "la configuración de esta red social, permite a los gestores de las páginas, activar o no, distintas opciones, encaminadas a recoger la opinión de los seguidores, tales como la posibilidad de enviar mensajes o la de ser puntuado en la escala del 1 al 5 (siendo 1 deficiente y 5 excelente)" Rodríguez (2017). Se ha encontrado, que ni en la página de Facebook del propio MAEC, ni la del consulado de Melbourne, ni la de la embajada de Egipto o Londres, tienen habilitada esta última opción y resulta imposible establecer comunicación vía redes sociales ya que, tampoco se permite realizar publicaciones, enviar mensajes o dar opiniones. En contraste, el consulado de Frankfurt tiene habilitada la opción de opiniones y publicaciones, aunque estas últimas reciban muy pocas respuestas. De las 10 publicaciones que se han encontrado en lo que va del 2018 (hasta julio) sólo 3 han recibido respuesta.

Así mismo, el consulado de Sao Paulo tiene habilitadas estas opciones y, en este caso, las respuestas también son escasas y en español, siendo que los mensajes han sido publicados en portugués.

Por su parte, de todos los mensajes enviados por los distintos canales que se proporcionan en las redes sociales (e-mails, mensajes privados, publicaciones u opiniones) consulados como el de Guadalajara en México y la embajada de Croacia cuentan en Facebook con un sistema de respuesta automatizada que pide inmediatamente que te comuniques vía email. En estos casos se enviaron los e-mails y solamente en el del Consulado General de Guadalajara en México, se recibió respuesta. Igualmente, la página de la Embajada de España en Australia cuenta con un sistema de respuesta automatizada. Aunque en este caso, el mensaje decía que en breve se atendería la petición. Posteriormente se recibió la respuesta oportuna.

Es de destacar que se recibió una respuesta inmediata a los mensajes directos, vía Facebook, por parte del Consulado de Lima, aunque ésta no respondía a la información que se estaba solicitando. Mismo caso sucede con la embajada de Ucrania, aunque tardaron más de 64 horas en responder. Las cuentas que no se mencionan es debido a que no se obtuvo ninguna contestación. 
En los casos de Twitter, todas las cuentas analizadas tenían la opción de mensajes directos bloqueados por lo que el único medio de contacto era twitteando a sus cuentas. No hubo respuesta a ninguno de los tweets enviados por la autora del presente estudio. En el caso de las cuentas analizadas de Instagram, se enviaron mensajes privados y tampoco hubo respuesta. Finalmente, vía YouTube no es posible enviar mensajes al servicio exterior español.

Con la presente investigación ha sido posible conocer que las cuentas en diferentes redes sociales del MAEC, consulados y embajadas españolas analizadas se han aperturado entre el 2010 y 2018. Siendo el 2015 año en el que más cuentas de la muestra se han creado.

Por otro lado, los responsables de la administración de estas redes sociales varían dependiendo de la embajada, consulado o el propio MAEC a la que pertenezcan. Careciendo, en casi todos los casos, de expertos especializados en comunicación digital o en redes sociales. Lo que se traduce, automáticamente, en una falta de profesionalización en lo que respecta al proceso de digitalización.

Con la ayuda de la herramienta Twitonomy, se efectuó una radiografía completa de las cuentas de Twitter analizadas en el presente estudio y a partir de ésta, ha sido posible obtener información valiosa.

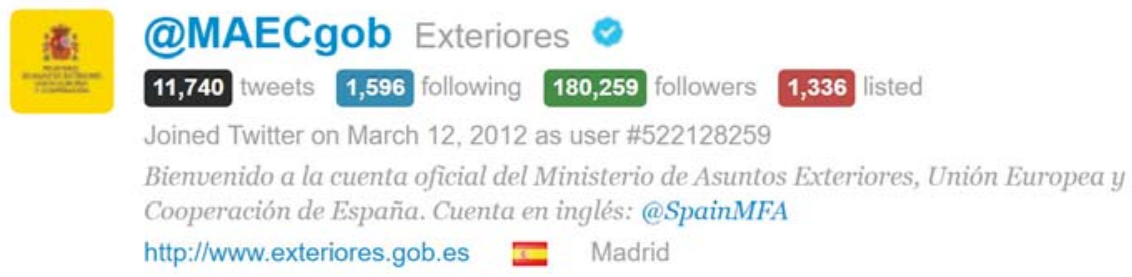

Imagen 2: Información básica de la cuenta de Twitter del MAEC. Fuente: Twitonomy.

A continuación, se incluyen el gráfico del historial de tweets publicados en la cuenta de Twitter del MAEC obtenido con Twitonomy. De este se puede observar que las publicaciones de la cuenta MAECgob son periódicas y constantes a lo largo de lo que va del 2018, con un promedio de 5,27 tweets diarios.

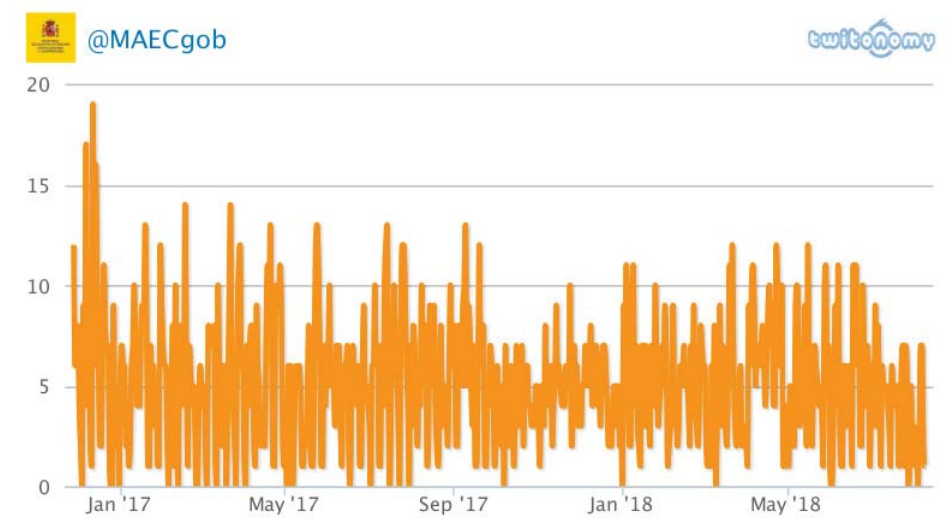

Gráfico 1: Historial de tweets publicados en la cuenta del MAEC de enero a julio del 2018. Fuente: Twitonomy 
En promedio, el $44 \%$ del contenido publicado por el servicio exterior español son retweets. Esta cantidad deberá disminuir si se busca incrementar la originalidad.

Se ratifica que el nivel de respuesta a las menciones recibidas en Twitter es bajísimo. Tan sólo un $4,71 \%$ de éstas se responden. Así mismo, se revela que el grado de engagement también es bajo. Solamente, un $19,45 \%$ de los tweets publicados en la muestra son retweetteados, y un $24,13 \%$ son marcados como favoritos por los usuarios.

Las cuentas de YouTube del MAEC, embajadas y consulados españoles son pocas, únicamente 9. Las visitas a éstas escasas, al igual que los subscriptores. Los contenidos aburridos e insuficientes.

Respecto a las cuentas de Instagram, es necesario que el servicio exterior español tome medidas para aprovechar al máximo los beneficios de esta red social relativamente nueva. Actualmente la cantidad de seguidores e interacción con éstas es reducida.

\begin{tabular}{|c|c|c|c|c|c|c|c|c|}
\hline \multicolumn{5}{|c|}{ Facebook } & \multicolumn{4}{|c|}{ Twitter } \\
\hline Cuentas & \begin{tabular}{|c|} 
Año en \\
creación
\end{tabular} & Puntuación (1-5) & $\begin{array}{c}\text { Publicaciones } \\
\text { (2018) }\end{array}$ & $\begin{array}{l}\text { Tiempo en } \\
\text { Respuesta }\end{array}$ & Cuentas & $\begin{array}{c}\text { Año en } \\
\text { creación }\end{array}$ & $\begin{array}{c}\text { Mensaje } \\
\text { privado }\end{array}$ & $\begin{array}{l}\text { Tiempo en } \\
\text { Respuesta }\end{array}$ \\
\hline MAEC & 2012 & No disponible & No disponible & $72 \mathrm{hr}$ & MAEC & 2012 & No disponible & Sin respuesta \\
\hline Consulado en Nueva York & 2015 & $4.8 / 30$ opiniones & \begin{tabular}{|l|}
8 publicaciones/ 0 \\
respuestas
\end{tabular} & $96 \mathrm{hr}$ & Consulado en Lyon & 2018 & No disponible & Sin respuesta \\
\hline Consulado en Lima & 2016 & 3.9/ 2 opiniones & $\begin{array}{l}2 \text { publicaciones/ } 0 \\
\text { respuestas }\end{array}$ & $1 \mathrm{hr}$ & Consulado en Houston & 2015 & No disponible & Sin respuesta \\
\hline Consulado en Guadalajara & 2016 & 4.5/ 17 opiniones & $\begin{array}{l}6 \text { publicaciones/ } 0 \\
\text { respuestas }\end{array}$ & Automatizada & $\begin{array}{l}\text { Consulado en } \\
\text { Jerusalen }\end{array}$ & 2014 & No disponible & Sin respuesta \\
\hline Consulado en Franckfurt & 2015 & No disponible & No disponible & Sin respuesta & $\begin{array}{l}\text { Embajada en Santa } \\
\text { Seen }\end{array}$ & 2018 & No disponible & Sin respuesta \\
\hline Consulado en Melbourne & 2010 & No disponible & No disponible & $24 \mathrm{hr}$ & $\begin{array}{l}\text { Embajada en } \\
\text { Kazajistán }\end{array}$ & 2015 & No disponible & Sin respuesta \\
\hline Consulado en Oran & 2015 & 3.8/ 34 opiniones & $\begin{array}{l}11 \text { publicaciones/ } \\
0 \text { respuestas }\end{array}$ & Sin respuesta & Embajada en Sudáfrica & 2015 & No disponible & Sin respuesta \\
\hline Consulado en Sao Paulo & 2014 & 4.4/ 29 opiniones & $\begin{array}{l}25 \text { publicaciones/ } \\
3 \text { respuestas }\end{array}$ & $2 \mathrm{hr}$ & & YouTu & abe & \\
\hline Consulado en Buenos Aires & 2015 & $3.7 / 38$ opiniones & $\mid \begin{array}{l}32 \text { publicaciones/ } \\
6 \text { respuestas }\end{array}$ & Sin respuesta & Cuentas & $\begin{array}{c}\text { Año en } \\
\text { creación }\end{array}$ & Subscriptores & Visitas \\
\hline Embajada en Riad & 2015 & 4.6/ 1 opinión & Disponibles & $\begin{array}{l}\text { Mensajes no } \\
\text { disponibles }\end{array}$ & MAEC & 2010 & 4,385 & 391,354 \\
\hline Embajada en Argentina & 2012 & $4.2 / 22$ opiniones & $\mid \begin{array}{l}4 \text { publicaciones/ } 0 \\
\text { respuestas }\end{array}$ & Sin respuesta & \begin{tabular}{|l|} 
Representación \\
permanente de España \\
en EU
\end{tabular} & 2017 & No disponible & No disponible \\
\hline Embajada en Croacia & 2014 & $4.8 / 3$ opiniones & $\begin{array}{l}3 \text { publicaciones/ } 1 \\
\text { respuesta }\end{array}$ & Automatizada & Embajada en Polonia & 2016 & No disponible & No disponible \\
\hline Embajada en Egipto & 2013 & No disponible & No disponible & Sin respuesta & () & Instagr & ram & \\
\hline Embajada en Pretoria & 2015 & No disponible & No disponible & Sin respuesta & Cuentas & $\begin{array}{c}\text { Año en } \\
\text { creación }\end{array}$ & $\begin{array}{c}\text { Tipo en } \\
\text { Mensajes }\end{array}$ & $\begin{array}{c}\text { Followers/ } \\
\text { Post }\end{array}$ \\
\hline Embajada en Australia & 2015 & No disponible & No disponible & Automatizada & MAEC & 2016 & $\begin{array}{r}\text { Privados/ Sin } \\
\text { respuesta }\end{array}$ & $8.595 / 323$ \\
\hline Embajada en Londres & 2017 & 3.5/ 8 opiniones & $\begin{array}{l}6 \text { publicaciones/ } 0 \\
\text { respuestas }\end{array}$ & Sin respuesta & Embajada en Singapur & 2016 & $\begin{array}{r}\text { Privados/ Sin } \\
\text { respuesta }\end{array}$ & $484 / 547$ \\
\hline Embajada en Ucrania & 2015 & 5/ 1 opinión & \begin{tabular}{|l|}
5 publicaciones/ 0 \\
respuestas
\end{tabular} & Sin respuesta & & & & \\
\hline
\end{tabular}

Tabla 2. Muestra y análisis de las redes sociales del servicio exterior español. (Fuente: creación propia)

\section{Diplomacia Digital Mexicana}

En el caso de México, en las últimas décadas su política exterior se ha caracterizado por un "periodo de incertidumbre en sus objetivos" Levy (2009) y por una falta 
de continuidad en los programas que se implementan, ya que desgraciadamente, el encargado principal de determinar cuál es el interés nacional, es el presidente de la Republica. Su mandato dura seis años y frecuentemente se encuentra que, cada vez que se cambia de mandatario, cambian los objetivos.

En el 2009, el entonces embajador mexicano en EE. UU., Arturo Sarukham, fué pionero en esgrimir Twitter como una herramienta de comunicación diplomática. Enfatizando la importancia de aprovechar la interconectividad de las sociedades contemporáneas para tener comunicación directa con los ciudadanos. Es decir, sin intermediarios, tales como los medios de comunicación o los gobiernos.

En 2013, en una entrevista realizada por Osvaldo Ojeda, el exembajador Arturo Sarukham sostuvo que toda cancillería moderna debe de tener como objetivo principal, capacitar en el uso de las redes sociales, tanto a los nuevos diplomáticos como a los más tradicionales.

Fruto de la llegada de Enrique Peña Nieto (EPN) a la presidencia, a mediados del 2013 se publica la "Estrategia Digital Nacional" en la cual queda recogido, como uno de los objetivos principales, alcanzar un gobierno más cercano, abierto y eficaz, ${ }^{7}$ reconociendo, de esta manera, que la digitalización del país es el camino correcto para lograr el desarrollo de la nación.

A raíz de la creación de la "Estrategia Digital Nacional" (EDN), la Secretaria de Relaciones Exteriores (SRE) creo un manual para el uso de redes sociales por parte de sus representantes en el exterior, en el cuál, se instaura la creación de cuentas oficiales de todas las misiones diplomáticas mexicanas en Facebook y en Twitter. En esta última, los titulares de dichas misiones también están obligados a tener presencia. Para mediados del 2016 las representaciones mexicanas contaban con 150 cuentas oficiales en Twitter. (Ver figura I)

\section{Figura I. Mapa de embajadas, consulados y misiones de México con cuentas de Twitter.}

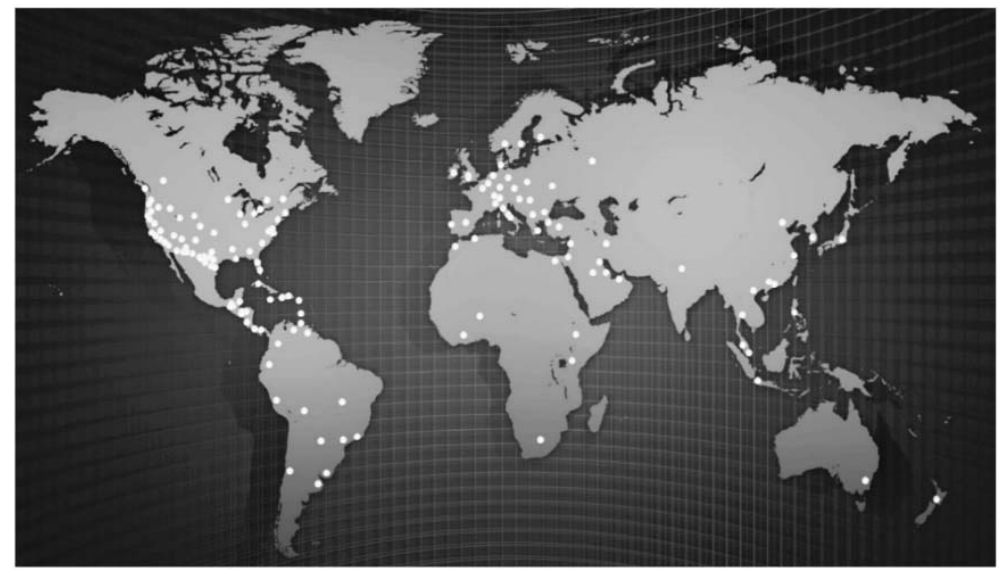

Fuente: “La diplomacia en 140 caracteres: El caso de México” Ramos (2018)

La "Estrategia Nacional Digital” Es un plan de acción gubernamental creado en el 2013 para incorporar las TIC en todas las áreas de la vida diaria de la sociedad, organizaciones y el gobierno, con esto se busca potenciar el desarrollo de México. 
En el manual mencionado anteriormente, "se establecen los contenidos que se deben publicar en las cuentas oficiales del servicio exterior mexicano" Casado (2017), basándose en los cinco pilares básicos de la diplomacia: representar, negociar, informar, proteger y promover los intereses de su Estado ante terceros, apoyando la mejora de la imagen del país. Información de México en el mundo, especialmente ante público extranjero. Fomentando el orgullo nacional y actividades de posicionamiento de la SRE. Con todo este conjunto de recomendaciones, se pretende incentivar la participación y el intercambio de opiniones entre y con los usuarios.

La Estrategia Digital Nacional y el manual creado por la SRE son una buena iniciativa del gobierno mexicano para continuar con el proceso de digitalización de la diplomacia mexicana. Dentro de la EDN se establecen estrategias y líneas de acción concretas entre las que destacan la promoción México a través de la difusión económica, turística y cultural mediante labores de la SRE. En resumen, se pretende consolidar la imagen del país, como un país de oportunidades, atractiva tanto para turistas como para inversionistas.

Para alcanzar las metas mencionadas anteriormente, se ha trabajado en la capacitación del personal diplomático relacionadas con la diplomacia pública y cultural tanto en el instituto nacional Matías Romero, como en instituciones internacionales, Méndez-Coto (2015). Es necesario hacer uso de todos los recursos humanos cualificados y que cuenten con la experiencia necesaria para desarrollar un proyecto de esta magnitud. Como, por ejemplo, el exembajador Arturo Surukham, reconocido diplomático a nivel internacional.

Por otro lado, pese al constante reconocimiento por parte del gobierno de la necesidad inminente de utilizar todas las tecnologías disponibles para la comunicación diplomática de México:

"Las redes sociales e internet se han convertido en los principales vehículos para la comunicación diplomática. Ello nos obliga a avanzar en la consolidación de una estrategia institucional de diplomacia digital que permita difundir contenidos de interés de forma efectiva y en tiempo real para un público diverso" 27 Reunión de Embajadores y Cónsules (2016)

Lamentablemente, la caída de la posición de México en el Ranking Global de la Diplomacia Digital del $2017\left(17^{\circ}\right)$ apunta a que las estrategias implementadas tienen fallos, ya que, en el mismo estudio del año 2016 , este país ocupó el puesto $\left(9^{\circ}\right)$. Aun así, México se encuentra en el tercer lugar a nivel América y en el segundo en lo que respecta a Iberoamérica.

Debido a que uno de los puntos clave de la diplomacia digital mexicana es incrementar la participación de la sociedad civil en temas de política exterior, la autora de este trabajo ha buscado obtener respuesta por parte del servicio exterior mexicano en sus plataformas digitales. En total se han enviado mensajes, hecho menciones o publicado comentarios en 17 perfiles de Facebook, 7 de Twitter, 3 de YouTube y 2 de Instagram. (Ver tabla 3)

Se ha descubierto que las cuentas de redes sociales del servicio exterior mexicano de la muestra fueron abiertas entre el año 2009 y el 2017, siendo el 2011 cuando más cuentas se crearon. En general, las cuentas de Twitter fueron las primeras en abrirse, seguidas por las de YouTube (en menor proporción), Facebook e Instagram. 

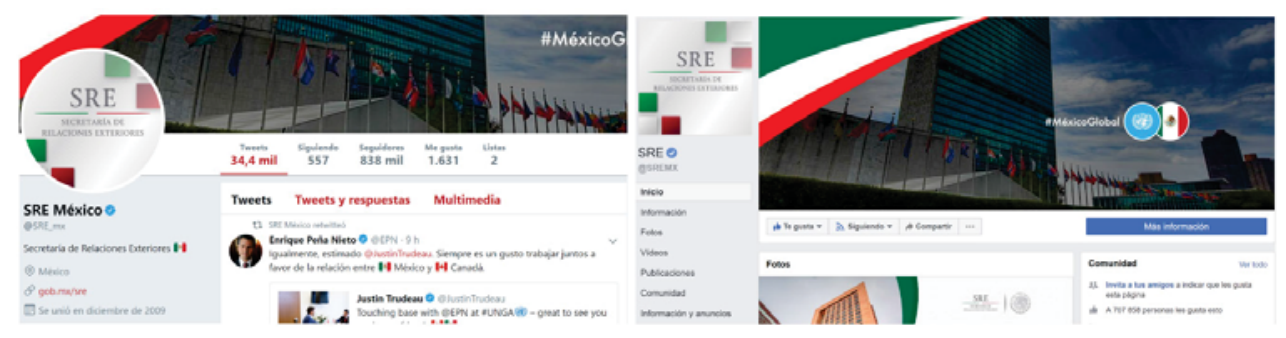

Imagen 3: Perfiles de las cuentas de Twitter (izquierda) y Facebook (derecha) de la SRE. Fuente: Cuentas de Twitter y Facebook de la SRE.

De todos los mensajes enviados, únicamente se recibieron 4 respuestas por medio de Facebook. Las cuales provenían de las embajadas de México en España, Estados Unidos, India y Qatar. En este aspecto, se concluye que los trabajadores del servicio exterior mexicano todavía no han sabido explotar todos los beneficios de las redes sociales. Omitir la interacción con su público es una falta grave que los aleja de alcanzar el máximo grado de la diplomacia digital.

$\mathrm{Al}$ igual que en el caso español, se efectuó una radiografía completa de las cuentas del servicio exterior mexicano de Twitter analizadas en el presente estudio. El primer descubrimiento consiste en que el contenido de la SRE, las embajadas y consulados publicado en sus cuentas de Twitter es propio, ya que en promedio tan solo un $38 \%$ de los tweets que se postean al día son retweets. Siendo la cuenta de la SRE la que menos retweets hace y la embajada de México en España la que más.

En la parte inferior se encuentra el gráfico del historial de tweets publicados en la cuenta de Twitter de la SRE obtenido con Twitonomy. Las publicaciones de la cuenta SRE_gob se realizan con frecuencia y varias veces al día. A lo largo de lo que va del 2018, tiene un promedio de 5,49 tweets publicados diariamentes.

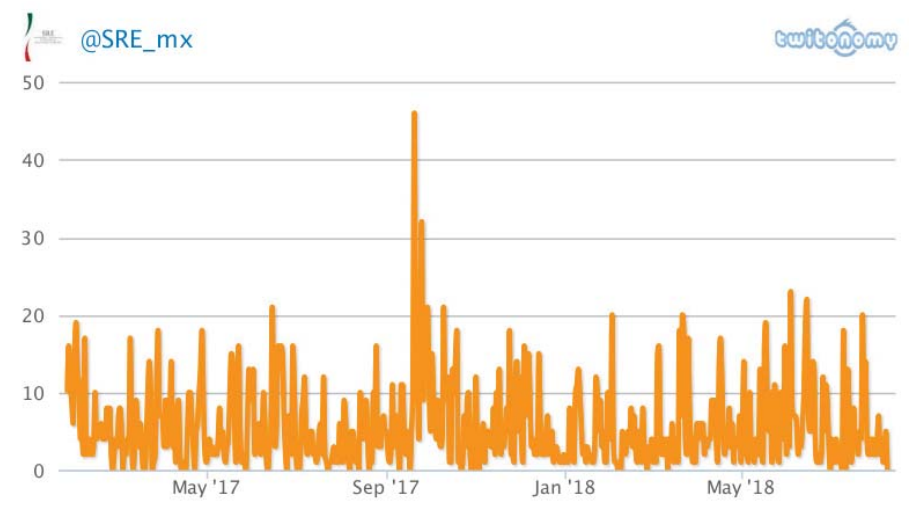

Gráfico 2: Historial de tweets publicados en la cuenta de la SRE de enero a julio del 2018. Fuente: Twitonomy. 


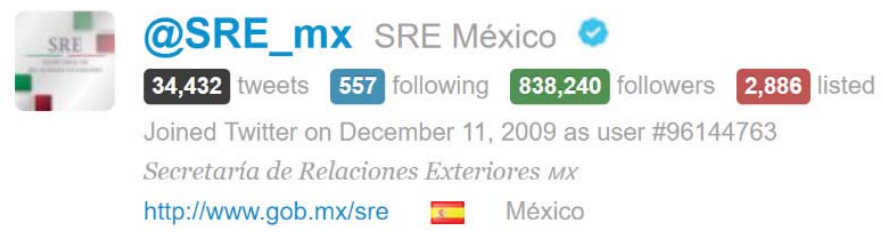

Imagen 4: Información básica de la cuenta de Twitter de la SRE. Fuente: Twitonomy.

A su vez, ha sido posible comprobar que las cuentas del servicio exterior mexicano de Twitter rara vez responden a las menciones que reciben. Tan sólo en un $0,57 \%$ de las ocasiones. A pesar de la falta de interacción que tienen los diplomáticos con los usuarios de redes sociales, se encontró que el engagement es alto, un promedio de $73,84 \%$ de los tweets analizados se retwittean y un $39,02 \%$ de ellos son marcados por los seguidores como favoritos.

En lo que respecta a YouTube, la SRE y sus representaciones en el exterior, poseen muy pocas cuentas. La cantidad de suscriptores y el número de visitas que reciben es escasa en comparación con otras redes sociales. Instagram es una red social que se está incorporando recientemente a los activos digitales diplomáticos mexicanos. A pesar del alto potencial que tiene para difundir mensajes, no se considera la mejor red social para interactuar con los ciudadanos.

Tabla 2. Muestra y análisis de las redes sociales del servicio exterior mexicano. (Fuente: creación propia)

\begin{tabular}{|c|c|c|c|c|c|c|c|c|}
\hline \multicolumn{5}{|c|}{ Facebook } & \multicolumn{4}{|c|}{ Twitter } \\
\hline Cuentas & \begin{tabular}{|c|} 
Año en \\
creación
\end{tabular} & Puntuación (1-5) & $\begin{array}{c}\text { Publicaciones } \\
(2018)\end{array}$ & $\begin{array}{l}\text { Tiempo en } \\
\text { Respuesta }\end{array}$ & Cuentas & \begin{tabular}{|c|} 
Año en \\
creación
\end{tabular} & $\begin{array}{c}\text { Mensaje } \\
\text { privado }\end{array}$ & $\begin{array}{l}\text { Tiempo en } \\
\text { Respuesta }\end{array}$ \\
\hline SRE_mx & 2012 & No disponible & No disponible & Sin respuesta & SRE_mx & 2009 & No disponible & Sin respuesta \\
\hline $\begin{array}{l}\text { Instituto de los Mexicanos } \\
\text { en el Exterior }\end{array}$ & 2017 & 5/ 4 opiniones & \begin{tabular}{|l|}
2 publicaciones/ 0 \\
respuestas
\end{tabular} & Sin respuesta & $\begin{array}{l}\text { Consulado en Hong } \\
\text { Kong }\end{array}$ & 2011 & No disponible & Sin respuesta \\
\hline Consulado en San Jose & 2014 & No disponible & No disponible & Sin respuesta & Consulado en Seattle & 2010 & No disponible & Sin respuesta \\
\hline Consulado en Milán & 2014 & $4.3 / 4$ opiniones & $\begin{array}{l}3 \text { publicaciones/ } 0 \\
\text { respuestas }\end{array}$ & Sin respuesta & $\begin{array}{l}\text { Consulado en San } \\
\text { Diego }\end{array}$ & 2011 & No disponible & Sin respuesta \\
\hline $\begin{array}{l}\text { Consulado en Río de } \\
\text { Janeiro }\end{array}$ & 2013 & No disponible & No disponible & Sin respuesta & Embajada en España & 2010 & No disponible & Sin respuesta \\
\hline Consulado en Hong Kong & 2015 & $4.5 / 3$ opiniones & \begin{tabular}{|l|}
2 publicaciones/ 1 \\
respuestas
\end{tabular} & Sin respuesta & Embajada en India & 2011 & No disponible & Sin respuesta \\
\hline Consulado en Atlanta & 2012 & 3.4/ 64 opiniones & \begin{tabular}{|l|}
64 publicaciones/ \\
8 respuestas
\end{tabular} & $72 \mathrm{hr}$ & Embajada en Brasil & 2011 & No disponible & Sin respuesta \\
\hline Consulado en Frankfurt & 2015 & No disponible & No disponible & Sin respuesta & & YouTu & abe & \\
\hline Embajada en España & 2011 & $3.7 / 38$ opiniones & $\begin{array}{l}32 \text { publicaciones/ } \\
6 \text { respuestas }\end{array}$ & $168 \mathrm{hr}$ & Cuentas & \begin{tabular}{|c|} 
Año en \\
creación
\end{tabular} & Subscriptores & Visitas \\
\hline Embajada en India & 2011 & 4.4/ 15 opiniones & \begin{tabular}{|l|}
13 publicaciones/ \\
0 respuestas
\end{tabular} & $64 \mathrm{hr}$ & SRE_mx & 2009 & 3,622 & $1,845,887$ \\
\hline $\begin{array}{l}\text { Embajada en Estados } \\
\text { Unidos }\end{array}$ & 2013 & $4.2 / 22$ opiniones & \begin{tabular}{|l|}
4 publicaciones/ 0 \\
respuestas
\end{tabular} & $30 \mathrm{hr}$ & Consulado en Detroit & 2013 & 154 & 34,226 \\
\hline Embajada en Ghana & 2015 & 5/ 1 opinión & $\begin{array}{l}1 \text { publicación/ } 0 \\
\text { respuesta }\end{array}$ & Sin respuesta & Embajada en España & 2010 & 551 & 335,339 \\
\hline Embajada en China & 2017 & $4.8 / 7$ opiniones & \begin{tabular}{|l|}
3 publicaciones/ 0 \\
respuesta
\end{tabular} & Sin respuesta & (0) & Instagr & ram & \\
\hline Embajada en Portugal & 2012 & $4.6 / 2$ opiniones & \begin{tabular}{|l}
2 publicaciones/ 0 \\
respuestas
\end{tabular} & Sin respuesta & Cuentas & \begin{tabular}{|c|} 
Año en \\
creación
\end{tabular} & $\begin{array}{c}\text { Tipo en } \\
\text { Mensajes }\end{array}$ & $\begin{array}{l}\text { Followers/ } \\
\text { Post }\end{array}$ \\
\hline Embajada en Qatar & 2014 & No disponible & No disponible & Sin respuesta & SRE_mx & 2015 & $\begin{array}{r}\text { Privados/ Sin } \\
\text { respuesta }\end{array}$ & $12 \mathrm{k} / 642$ \\
\hline Embajada en Ecuador & 2014 & No disponible & No disponible & Sin respuesta & Embajada en Irán & 2017 & $\begin{array}{r}\text { Privados/ Sin } \\
\text { respuesta }\end{array}$ & $231 / 388$ \\
\hline Embajada en Reino Unido & 2014 & $4.2 / 33$ opiniones & \begin{tabular}{|l|}
7 publicaciones/ 0 \\
respuestas
\end{tabular} & Sin respuesta & & & & \\
\hline
\end{tabular}




\section{Comparativa de los procesos de digitalización de España y México}

\begin{tabular}{|c|c|c|}
\hline Tema & España & México \\
\hline $\begin{array}{l}\text { Estrategia } \\
\text { de Digitalización }\end{array}$ & $\begin{array}{l}\text { *Normas básicas de comportamiento } \\
\text { en las Redes Sociales para las } \\
\text { Representaciones de España en el } \\
\text { Exterior (2011) } \\
\text { *Plan de Diplomacia Digital (2012) } \\
\text { *Reglamento de la Carrera Diplomática } \\
\text { (2014) } \\
\text { *Plan de Comunicación de las Misiones } \\
\text { Diplomáticas, Representaciones } \\
\text { Permanentes y Oficinas Consulares de } \\
\text { España en el Exterior (2016) }\end{array}$ & $\begin{array}{l}\text { *Plan Nacional de Desarrollo (2013- } \\
\text { 2018) } \\
\text { *Estrategia Digital Nacional (2013) } \\
\text { *Manual para el Uso de las Redes } \\
\text { Sociales por las Representaciones } \\
\text { Mexicanas en el Exterior (2013) } \\
\text { *XXVII Reunión de Embajadores y } \\
\text { Cónsules (2016) }\end{array}$ \\
\hline * Ventajas & $\begin{array}{l}\text { *Mejora de la navegabilidad del sitio } \\
\text { web del MAEC. } \\
\text { *Cercanía e interlocución con los actores } \\
\text { internacionales y nacionales. } \\
\text { *Nombramiento de un embajador digital. } \\
\text { *Programa de formación digital. } \\
\text { *Embajadas "hub" } \\
\text { *Aumento de la transparencia. } \\
\text { *Democratización de la acción exterior. } \\
\text { *Uso del teléfono móvil para enviar } \\
\text { información básica de los consulados. }\end{array}$ & $\begin{array}{l}\text { *Acercamiento a la } \\
\text { ciudadanía mexicana. } \\
\text { *Proyectar una imagen positiva de } \\
\text { México en el extranjero. } \\
\text { *Herramientas SREMonitor (una } \\
\text { app que segmenta la información por } \\
\text { temas de interés) y SREtv (canal para } \\
\text { transmitir contenidos). } \\
\text { *Suficiente infraestructura digital. }\end{array}$ \\
\hline$*$ Retos & $\begin{array}{l}\text { *Falta de presupuesto. } \\
\text { *Limitaciones institucionales. } \\
\text { *Homologación del proceso de } \\
\text { digitalización del MAEC. } \\
\text { *Discriminación de la comunicación. } \\
\text { *Manejo de las relaciones con un mayor } \\
\text { número de actores. } \\
\text { *Modernización de los tramites y acceso } \\
\text { a la documentación. } \\
\text { *Garantizar la seguridad y privacidad de } \\
\text { la información. } \\
\text { *Incentivar la formación continuada. } \\
\text { *Atención en casos de emergencia. } \\
\text { *Incrementar la escucha e interacción } \\
\text { con los usuarios. }\end{array}$ & $\begin{array}{l}\text { *Incrementar la escucha e interacción } \\
\text { con los usuarios. } \\
\text { *Alcanzar la cultura de datos abiertos } \\
\text { que se busca. } \\
\text { *Homologación de los procesos de } \\
\text { acción y respuesta en lo que respecta al } \\
\text { terreno digital. } \\
\text { *Garantizar la seguridad y privacidad de } \\
\text { la información. } \\
\text { *Incentivar la formación continuada. } \\
\text { *Atención en casos de emergencia. }\end{array}$ \\
\hline $\begin{array}{l}\text { Presencia } \\
\text { en Redes Sociales }\end{array}$ & $\begin{array}{l}\text { Twitter, Facebook, Instagram, YouTube, } \\
\text { Weibo y Vkontakte. }\end{array}$ & $\begin{array}{l}\text { Twitter, Facebook, Instagram, YouTube } \\
\text { y Weibo. Puede tener presencia en más } \\
\text { redes sociales, el problema es que no hay } \\
\text { acceso público a esa información. }\end{array}$ \\
\hline $\begin{array}{l}\text { Responsable(s) } \\
\text { de la Digitalización }\end{array}$ & Cónsul, funcionarios. & $\begin{array}{l}\text { En general por } 1 \text { o } 2 \text { funcionarios } \\
\text { miembros del servicio exterior o locales } \\
\text { dedicados a brindar comunicados de } \\
\text { prensa. }\end{array}$ \\
\hline $\begin{array}{l}\text { Porcentaje de respuesta en } \\
\text { Facebook }\end{array}$ & $52,94 \%$ & $23,52 \%$ \\
\hline $\begin{array}{l}\text { Aproximado de } \\
\text { respuestas automatizadas } \\
\text { en Facebook }(\%)\end{array}$ & $17,64 \%$ & $11,76 \%$ \\
\hline
\end{tabular}




\begin{tabular}{|l|c|c|}
\hline $\begin{array}{l}\text { Promedio de la puntuación } \\
\text { en Facebook (1-5) }\end{array}$ & $3.91^{8}$ & $4.02^{9}$ \\
\hline Respuesta en Twitter & $4,71 \%$ & $0,57 \%$ \\
\hline Tweets Retweeteados (\%) & $19,45 \%$ & $73,84 \%$ \\
\hline Tweets Favoritos (\%) & $24,13 \%$ & $39,02 \%$ \\
\hline
\end{tabular}

En la comparativa realizada en la tabla superior se observa que ninguno de los países estudiados cuenta con personal encargado exclusivamente de los procesos de digitalización y manejo de las redes sociales, webs y blogs, lo que se traduce automáticamente en la ausencia de respuestas oportunas y rápidas a las inquietudes, preguntas o comentarios de los usuarios. En suma, se ha recibido una mayor cantidad de respuestas a través de Facebook por parte del servicio diplomático español que del mexicano, que más bien ha sido nulo. Por su parte, en la red social Twitter, el porcentaje de respuesta de ambos países es extremadamente bajo, en el caso español un $4,71 \%$ de los usuarios reciben respuesta, y en el caso mexicano solo un $0,57 \%$.

En lo que respecta al uso de Twitter, el gobierno mexicano ha sabido hacer un mejor uso de esta red social, logrando tener más potencial de influenciabilidad. Esto se observa claramente con la cantidad de retweets que reciben las publicaciones del servicio exterior mexicano, alcanzando un $73,84 \%$, y un $39,02 \%$ son marcados como favoritos por los usuarios. Por el contrario, se aprecia que el gobierno español tiene menos alcance en esta red social, tan solo el $19,45 \%$ de sus tweets son retweeteados por los usuarios y un $24,13 \%$ de estos son marcados como favoritos.

\section{Conclusiones}

Gobiernos hispanoamericanos, como el español y el mexicano, reconocen la necesidad de incorporar las TIC a su día a día mediante la creación de estrategias de digitalización que afectan a sus respectivas administraciones, incluyendo sus servicios exteriores.

En base a los resultados obtenidos del presente estudio podemos concluir que pese a que en los últimos diez años los servicios exteriores de España y México han logrado tener presencia en las principales redes sociales (Twitter, Facebook, YouTube, Instagram, entre otras) aún es posible encontrar brechas en lo que respecta a la actualización de los contenidos, interacción con el público, influencia y por supuesto autorrealización.

Innegable es que, para llevar a cabo una migración total de lo analógico a lo digital, contar con personal cualificado y capacitado en los servicios exteriores es indispensable. El reto consiste en concientizar a diplomáticos tradicionales de que el mundo ha cambiado drásticamente, lo que antes era conocido como información confidencial, se ha convertido en información de dominio público y deben aprender a manejar esta nueva situación. Los servicios exteriores deben proveer de los entrenamientos y cursos necesarios a sus trabajadores, permitiéndoles desempeñarse con confianza en sus puestos y realizar las actividades indispensables para cumplir sus objetivos en esta era digital.

Solo se han tomado en cuenta las puntuaciones de la muestra que tenían habilitada esta opción (11)

Solo se han tomado en cuenta las puntuaciones de la muestra que tenían habilitada esta opción (11) 
Por otra parte, es forzosa una homologación de los procedimientos y metodologías de trabajo en las redes sociales y otras herramientas de comunicación de los ministerios/ secretarias, embajadas y consulados para alcanzar el grado de digitalización anhelado. Actualmente, los servicios exteriores de España y México utilizan las redes sociales para compartir contenidos que consideran oportunos e información útil para ciudadanos nacionales e internacionales, sin embargo, la frecuencia de las publicaciones y el tipo de contenido es desigual dependiendo de a donde pertenezcan.

El presente estudio permite afirmar, al igual que lo hizo Andrés Rodríguez refiriéndose a las redes sociales de la administración española, que hablando de los servicios exteriores de México y de España "el nivel de respuesta a los ciudadanos es muy bajo y la realidad es que estos perfiles apenas son usados para interactuar con la opinión pública y favorecer la escucha activa, el debate, la participación y la colaboración”, Rodríguez (2017).

\section{Bibliografía}

Banda, Vianney. (2015). La diplomacia en tiempos de las redes sociales. Blog \#SocialMediaAcatlán. Recuperado en 21 de julio de 2018, de http://blogs.acatlan.unam.mx/smacatlan/2015/06/16/la-diplomacia-en-los-tiempos-de-las-redes-sociales/

Bjola, Corneliu y Lu Jiang. (2015). Social Media and Public Diplomacy. A comparative analysis of the digital diplomatic strategies of the EU, US and Japan in China. En Bjola, Corneliu y Marcus Holmes (Eds.), Digital Diplomacy, Theory and Practice (pp. 89-108). Nueva York: Routledge.

Ott, Brian L. (2017) The age of Twitter: Donald J. Trump and the politics of debasement, Critical Studies in Media Communication, 34:1, 59-68, DOI: 10.1080/15295036.2016.1266686, Recuperado en 15 de julio de 2018, de https://rws511.pbworks.com/w/file/ fetch/119836821/Ott\%20The\%20age\%20of\%20Twitter\%20and\%20Trump.pdf

Cartmell, Matt. (2008) Foreign Office goes live with $20 \mathrm{~m}$ pounds integrated site. PRWeek Regions. Recuperado en 27 de julio de 2018, de https://www.prweek.com/article/799593/ foreign-office-goes-live-20m-integrated-site

Casado, R., Antonio. (2017). "Diplomacia 3.0": de la comunicación digital a la diplomacia digital. Análisis, N9, Recuperado en 24 de julio de 2018, de http:/www.exteriores.gob.es/ Portal/es/SalaDePrensa/Multimedia/Publicaciones/Documents/2017_\%20ANALISIS_9. pdf e

Chounet-Cambas, Anne. (2014) Manos en el motor de la diplomacia digital. Carnets diplomatiques Recuperado en 27 de julio de 2018, de http://blog.diplomatie.gouv.fr/Lesmains-dans-le-moteur-de-la?lang=fr

Cortés, Antón. (2016). La diplomacia española ante el reto digital. España: MAEC 2016 Libro Diplomacia Digital

Corujo, Adolfo. (s/f). El Facebook del embajador o el uso de las redes sociales en las relaciones diplomáticas. Desarrollo Ideas Llorente\&Cuenca, Revista UNO, No 17. Recuperado en 27 de julio de 2018, de https://www.revista-uno.com/numero-17/el-facebook-del-embajador-o-el-uso-de-las-redes-sociales-en-las-relaciones-diplomaticas/

France Diplomatie. (2017). La estrategia internacional de Francia para las tecnologías digitales. Recuperado en 27 de julio de 2018 de https://www.diplomatie.gouv.fr/es/asun- 
tos-globales/diplomacia-digital/diplomacia-de-influencia-digital/article/digital-la-estrategia-internacional-de-francia-para-las-tecnologias-digitales

Gobierno de la República. (2013). Estrategia Digital Nacional. Recuperado en 27 de julio de 2018 de https://www.gob.mx/cms/uploads/ attachment/file/17083/Estrategia_Digital_Nacional.pdf

Hugh, Elliot. (2017) Digital Diplomacy: are we the champions? Foreign and Commonwealth Office Recuperado en 27 de julio de 2018, de https://blogs.fco.gov.uk/guestpost/2017/03/30/digital-diplomacy-are-we-the-champions/

Lee, Matthew. (2009) Hillary Clinton, e-diplomat, embraces new media. The Sydney Morning Herald Recuperado el 26 de julio de 2018, de https:/www.smh.com.au/technology/ hillary-clinton-ediplomat-embraces-new-media-20090324-97nh.html

Levy, Carlos. (2009). Crisis y retos de la política exterior de México: 2006-2012. Revista mexicana de ciencias políticas y sociales, 51(205), 119-141. Recuperado en 24 de julio de 2018, de http://www.scielo.org.mx/scielo.php?script=sci_arttext\&pi$\mathrm{d}=\mathrm{S} 0185-19182009000100007 \& \operatorname{lng}=$ es\&tlng=es.

Manfredi, Juan, L. (2016). La transformación profesional de la diplomacia en red. En J. L. Manfredi y C. Femenía (Eds.), La diplomacia española ante el reto digital (pp. 101-110). Madrid: Ministerio de Asuntos Exteriores y Cooperación.

Manfredi, J. L., Rubio, R. y Pérez de Ágreda, E. (2015). La diplomacia pública como reto de la política exterior. Madrid, Ministerio de Asuntos Exteriores y Cooperación.

Manfredi, Juan, L. (2014). El desafío de la diplomacia digital. Blog Real Instituto Elcano. Recuperado en 21 de julio de 2018 de, http:// www.realinstitutoelcano.org/wps/portal/ web/rielcano_es/contenido?WCM_GLOBAL_CONTEXT=/elcano/elcano_es/zonas_es/ politicaexteriorespanola/ari152014-manfredi-desafio-diplomacia-digita

Murray, Stuart. (2015) The digital divide in American and Australian contexts en Digital Diplomacy: Theory and Practice. Routledge, New York. PP. 132

Méndez-Coto, Marco, et. (2016). Diplomacia pública y marca-país en las Américas: Un acercamiento a las experiencias de Brasil, Chile, México y Perú Temas de nuestra América, 32(59), 81-98. Recuperado en 24 de julio de 2018, de https:/www.researchgate.net/publication/303957638_Diplomacia_publica_y_marca-pais_en_las_Americas_Un_acercamiento_a_las_experiencias_de_Brasil_Mexico_y_Peru

Parra, Camilo. (s/n). El engagement es como una relación amorosa. Marketing de contenidos. Recuperado en 24 de julio de 2018, de https://marketingdecontenidos.com/que-es-engagement/

Rubio, R. (2011). Diplomacia digital. Una introducción. Madrid, Cuadernos de la Escuela Diplomática. Recuperado de https:/www.academia.edu/5685126/Diplomacia_digital. Una_introducción.Las_relaciones_internacionales_en_el_tránsito_al_siglo_XXI http:// www.rafarubio.es/wp-content/uploads/ciberdiplomaciaintro.pdf

Ramos, C., Alejandro et Espinoza, C., Luz. (2018). La diplomacia en 140 caracteres: El caso de México. en Diplomacia Pública Digital: El contexto Iberoamericano, en Escuela de Relaciones Internacionales de la Universidad Nacional, pp 33- 70, Recuperado en 23 de julio de 2018, de http://www.repositorio.una.ac.cr/bitstream/handle/11056/14356/publication.pdf? sequence $=1$ \&isAllowed $=\mathrm{y}$

Rodríguez, Alfredo, A. (2013). La diplomacia pública española desde 1939 a 2012. Comunicación, imagen y marca España. Universidad Complutense de Madrid. Madrid. Recuperado en 20 de julio de 2018, de https:/eprints.ucm.es/25350/1/T35343.pdf

Rodríguez, Alfredo, A. (2015). Diplomacia digital, ¿adaptación al mundo digital o nuevo modelo de diplomacia? Opción, vol. 31, núm. 2, pp. 915-937. Universidad del Zulia. 
Maracaibo, Venezuela. Recuperado en 23 de julio de 2018, de http://www.redalyc.org/ pdf/310/31045568050.pdf

Rodríguez, Andrés, R. (2017). «¿En camino hacia el gobierno 2.0?: análisis del uso de redes sociales por los ministerios españoles para fomentar la transparencia y la participación ciudadana», en Estudios sobre el Mensaje Periodístico 23 (2), 937-954.

Sarukham, Arturo. (2016). Intervención en el Seminario internacional México Global: intereses y principios de política exterior. Recuperado de https://www.youtube. com/watch?$\mathrm{v}=\mathrm{kdrxVXGKDs} 8$

Sotiriu, Sabrina. (2015). Digital Diplomacy. Between Promises and Reality. En Bjola, Corneliu y Marcus Holmes (Eds.), Digital Diplomacy, Theory and Practice (pp. 4666). Nueva York: Routledge.

Sharma, Dave. (2015) Digital Diplomacy: Making Friends in the Age of Facebook. Tablet Magazine Recuperado el 26 de julio de 2018, de https://www.tabletmag.com/jewish-news-and-politics/192177/digital-diplomacy/?print=1 